\title{
IRAS 18456-0223 - a flare star in a new star forming region ${ }^{\star}$
}

\author{
S. Kimeswenger and R. Weinberger
}

\author{
Institut für Astrophysik der Leopold-Franzens-Universität Innsbruck, Technikerstraße 25, 6020 Innsbruck, Austria \\ http://astro.uibk.ac.at
}

Received 11 January 2001 / Accepted 16 February 2001

\begin{abstract}
We report the discovery of a new conspicuous flare star coincident with the position of the IRAS PSC source 18456-0223 that is located in or projected on a dark cloud. In the immediate vicinity of the source, a very faint nebula and a small group of stars are detected in the optical. We carried out rough red band photometry and accurate astrometry of those stellar objects. In addition, near-infrared images were obtained with the DENIS survey instrument in $I(0.8), J(1.25)$ and $K_{\mathrm{s}}(2.15 \mu \mathrm{m})$ that show an increasing number of stars which appear to constitute a small cluster around the position of the IRAS source; the flare star itself $\left(R_{\mathrm{MAX}} \approx 15.55, R_{\mathrm{MIN}}>\right.$ $22^{\mathrm{m}} 5$ ) however, could not be traced. We assume that the flare star is enshrouded in a dust cloud of $A_{V}>10^{\mathrm{m}}$. By using a $K$ vs. $J-K$ diagram we were able to detect a new star forming region around IRAS 18456-0223 by identifying a number of YSO candidates. The distance estimate is $1600 \pm 400 \mathrm{pc}$ and that of the foreground extinction $A_{V}=2 \pm 0.5$ mag.
\end{abstract}

Key words. surveys - stars: flare - stars: formation - ISM: clouds

\section{Introduction}

Although made half a century ago, the Palomar Observatory Sky Survey (POSS I) has still not run out of its richnesses. Members of our institute have carried out several systematical surveys on POSS I and have detected thousands of various objects, among them primarily galaxies in the zone of avoidance, and planetary nebulae (e.g. Saurer et al. 1997; Hartl \& Weinberger 1987). As a by-product or extension of these searches we also came upon conspicuous variable objects found on POSS I overlapping regions or by comparison with other optical surveys (e.g. Weinberger 1991). One of our hitherto unstudied variable candidate stars is an object visible on a POSS I red-sensitive print located in or projected on a dark cloud, but invisible on any other survey plates. We believe that we deal with a new flare star candidate.

Flare stars - yellow or red dwarfs of low luminosity and age - sporadically brighten by typically $\sim 1^{\mathrm{m}}$ over (tens of) minutes (Ambartsumian 1990). The candidate under discussion appears to have brightened by $>6-7 \mathrm{mag}$. Based on DENIS data, we have identified in this area a subregion of a star forming cloud as yet unregistered.

Send offprint requests to: S. Kimeswenger, e-mail: Stefan.Kimeswenger@uibk.ac.at

* Based on observations collected at the European Southern Observatory, La Silla, Chile at the DENIS consortium survey instrument.

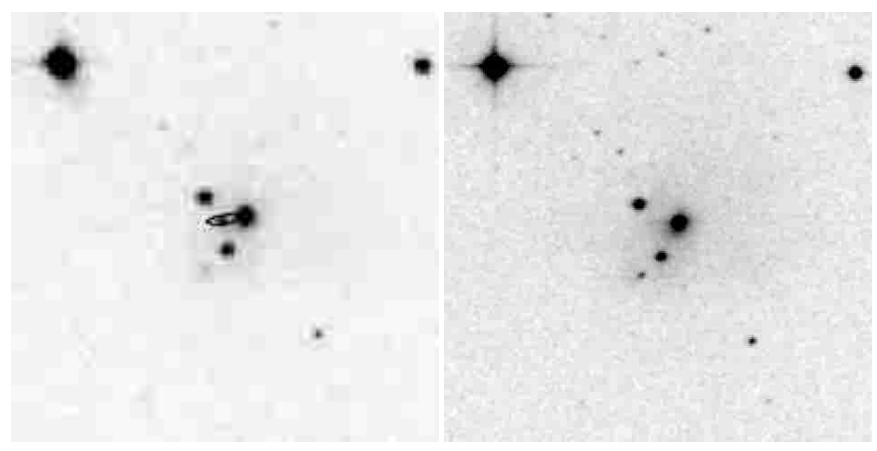

Fig. 1. A reproduction $\left(4^{\prime} \times 4^{\prime}\right)$ from the POSS I E plate No. 193 (left) and the POSS II R No. 879 plate (right). The flare star, clearly visible in the center of a small star group, coincides with an IRAS PSC source, whose positional error ellipse is shown

\section{The data}

The flare star candidate (Fig. 1) was detected on the red-sensitive POSS I plate E193 $\left(\lambda_{\text {eff }} \approx 645 \mathrm{~nm}\right)$ taken 50 years ago (1950.546). To estimate its brightness we used some USNO A2 stars listed in Table 1 (Monet et al. 1998).

The presence of both a very faint nebula and the star group (Fig. 1) reduces somewhat the accuracy; we estimate a red magnitude of $15.55 \pm 0.3$. The candidate is visible only on POSS I E193 but not on its blue-sensitive counterpart O193. It is also absent on all other deep sky survey plates (like POSS II plates) with their limiting magnitude of up to $\sim 22.5 \mathrm{mag}$ or plates of the Near Infrared 
Table 1. USNO A2 stars used for calibrating the red POSS I plate

\begin{tabular}{lccl}
\hline Identification & $m_{\text {blue }}$ & $m_{\text {red }}$ & Remark \\
\hline $0825-13050151$ & 17.6 & 15.4 & \\
$0825-13060719$ & 17.6 & 14.8 & \\
$0825-13033689$ & 15.0 & 12.9 & \\
$0825-13050693$ & 18.7 & 15.6 & \\
$0825-13034917$ & 18.5 & 15.9 & \\
$0825-13045391$ & 16.5 & 14.6 & \\
$0825-13044642$ & 16.9 & 13.3 & box in Fig. 2 \\
\hline
\end{tabular}

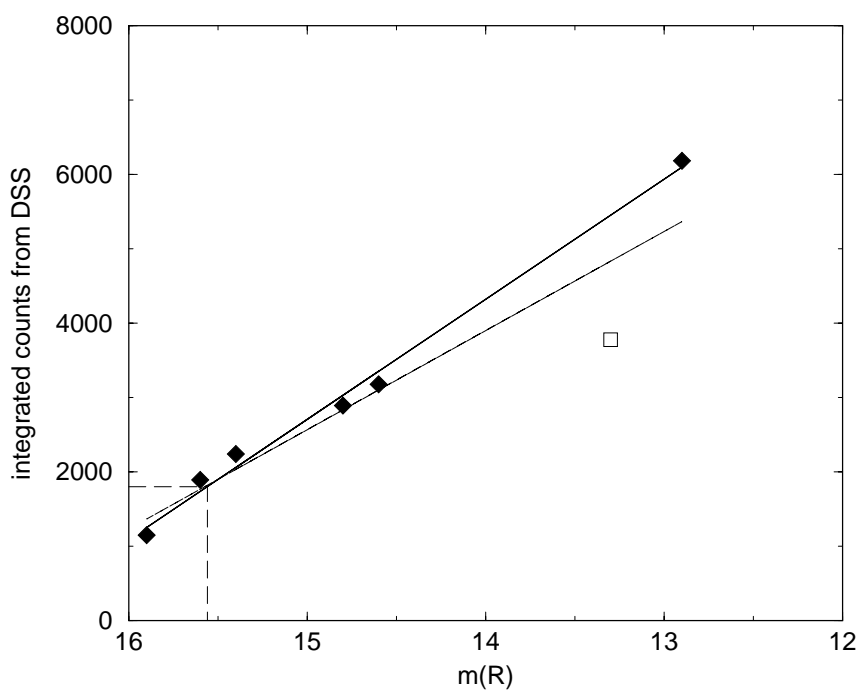

Fig. 2. The photometry of the flare star candidate using aperture photometry of the stars listed in Table 1. The thick line is the regression excluding the star marked with an open box. The thin line does include this star in the regression. The value obtained for the target is marked. The star 0825-13044642 is fainter than 0825-13045391 on all sky survey plates and on all CCD frames. Thus we assume that the USNO automated magnitude was faked by the nearby bright object $0825-13043990$ and the underlying optical nebulosity

Photographic Survey by Hoessel et al. (1979). In 1996/97 several CCD frames at the Innsbruck $60 \mathrm{~cm}$ telescope were obtained in $I$ and $R$. The limiting magnitudes are comparable to those of the corresponding sky survey plates ( 19-20 mag), but does not show the flare star candidate either.

Since we could, a priori, not entirely exclude that the flare star candidate is rather a roundish plate defect than a real object (it looks, however, quite like a star under the microscope), we verified its nature via its point spread function (PSF) using a mean PSF. To this end we extracted the images of all other non-blended stars of comparable brightness within a radius of $10^{\prime}$. These images where normalized to an integrated flux of 1.0 and centered (Gaussian center). Then the mean of the 1 images was derived. The shape is, as expected, not identical to a pure Gaussian (flat top). This mean PSF was scaled to the height of the flare star and then subtracted. As the
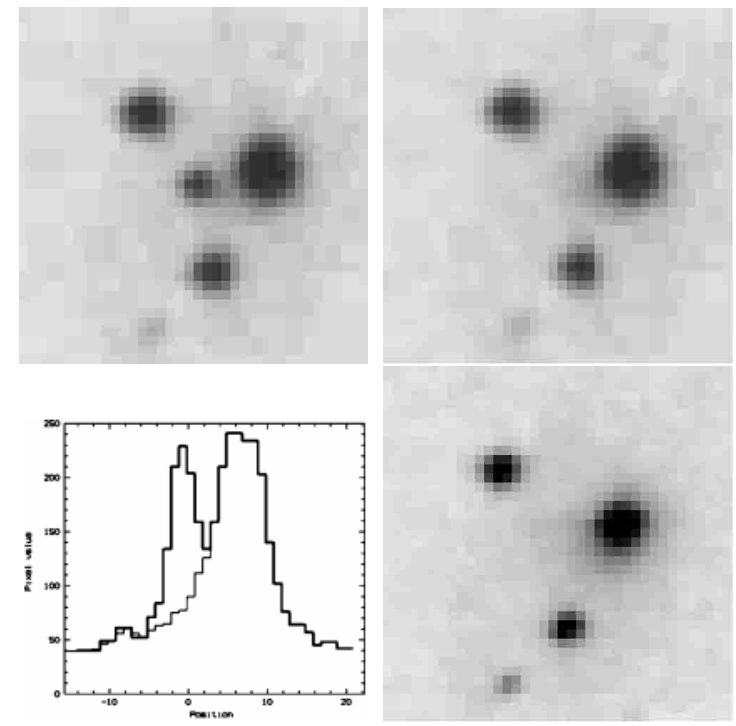

Fig. 3. The star group containing the flare star candidate taken from the POSS I E plate before (upper left) and after the subtraction of the mean PSF of a star (upper right). The nebulosity which remains under the stellar position is real as the POSS II R plate (lower right) shows. The graph displays the E-W scan through the image before (thick line) and after (thin line) the procedure

center was fixed by fitting, the scaling height was the only degree of freedom in this procedure. The results are given in Fig. 3. Thus we may safely conclude that the candidate is indeed a real object - a star that underwent a strong optical flare 5 decades ago.

Interestingly, the position of the flare star coincides exactly with an IRAS point source. To demonstrate this coincidence a precise determination of the position of the star is required of course, and we carried out astrometry using scanned images of POSS I E: The three visually brightest stars of the central group and the star towards the lower right corner are (from north to south) the USNO A2 stars 0825-13045391, 0825-13043990, 0825-13044642 and 0825-130414457, respectively. The bright star in the upper left corner of Fig. 1 is HD 173955 and is included in the TYCHO2 catalogue. The star 0825-13043990 seems to be affected on the scans and on the DENIS $I$ and $J$ images by a very faint nebula and thus was "elongated". We therefore derived the rms noise in those channels excluding this star. In the $K$ band we were able to use all stars. The individual errors are about 0 .' 4 in both coordinates. A crosscheck with the TYCHO2 coordinates gave no hint to a systematic shift of the coordinate systems. Thus we derived

$$
\begin{array}{ll}
\alpha=18^{\mathrm{h}} 48^{\mathrm{m}} 16^{\mathrm{s}} \cdot 23_{ \pm 0.03} & (\mathrm{~J} 2000) \\
\delta=-02^{\circ} 20^{\prime} 14^{\prime \prime} 8_{ \pm 0.5} & (\mathrm{~J} 2000)
\end{array}
$$

for the flare star. Although the star USNO A2 0825-13043990 is located at the very border of the IRAS positional error ellipse, we assume, due to the unusual nature and the exact coincidence, that the flare star is physically associated to the IRAS PSC source 18456-0223. 


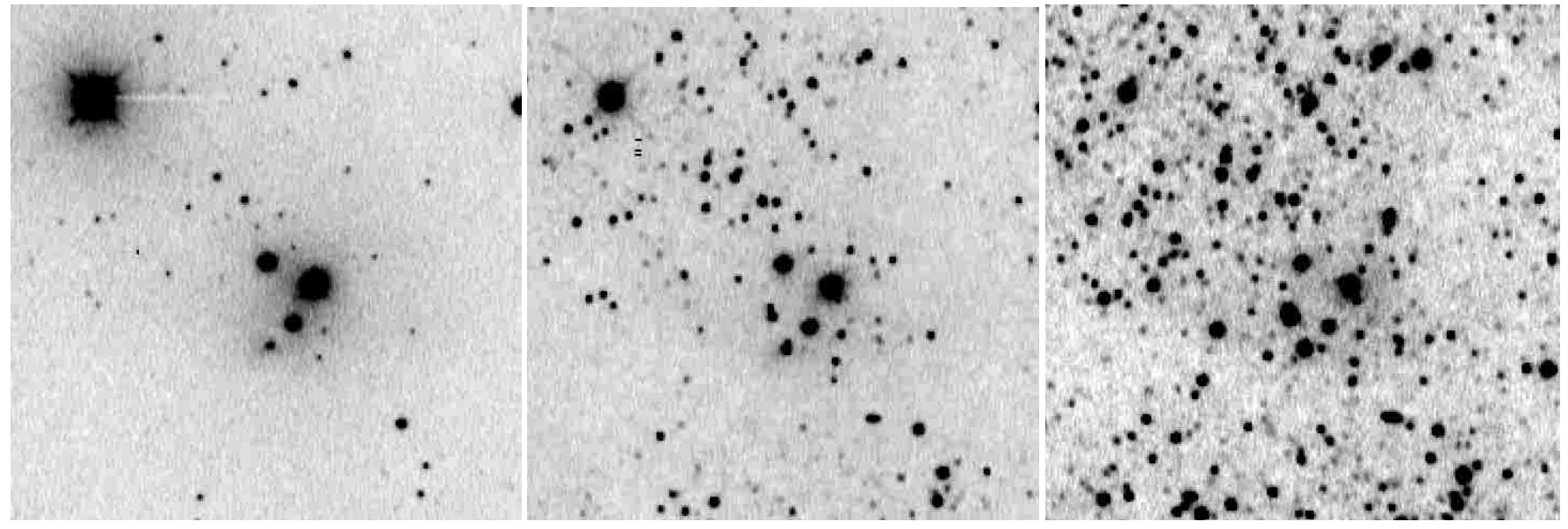

Fig. 4. A stack of 10 DENIS NIR images $\left(I, J, K_{\mathrm{s}}\right.$ from left to right) of the region around IRAS 18456-0223. The stellar limiting magnitudes are $19.60,18.00$ and 15.45 , respectively

This source was detected in 12 and $25 \mu \mathrm{m}$ only. The 60 and $100 \mu \mathrm{m}$ wavelengths are overlaid by a bright background component, which is most likely originating from cold dust in the cloud itself (see also Fig. 6). There is no detection of a $6.7 \mathrm{GHz}$ methanol maser emission by a survey arround selected IRAS sources (Szymczak et al. 2000). The IRAS LRS spectrum was classified by Kwok et al. (1997) as $P$ (= blue peaking with extended red excess) which is common for embedded stellar sources.

Near infrared (NIR) data were obtained using the DENIS survey instrument at the ESO $1 \mathrm{~m}$ telescope (Epchtein et al. 1997; Kimeswenger et al. 1998) on June 22th, 2000, 6:18 UT. The images were taken simultaneously in all three bands, Gunn- $I(0.82 \mu \mathrm{m}), J$ $(1.2 \mu \mathrm{m})$ and $K_{\mathrm{s}}(2.15 \mu \mathrm{m})$. Each band was observed 10 times while moving around the source in the field of view. This was done to eliminate errors due to local flatfield effects and in order to obtain the sky background using the iso-airmass median sky filtering. The procedure provides also an estimate of the intrinsic error of the measurement, being better than 0.05 for stars more than $3 \sigma$ above the background and better than 0.03 mag for stars above a $5 \sigma$ level. The flux calibration was done individually using all standards observed during the night. We did not use the extinction correction applied in general for the DENIS survey (Epchtein et al. 1999; Fouqué et al. 2000) but derived it individually for all three bands. Finally all 10 images were combined. The limiting magnitudes (well above noise) of those stacked images are about 19.60, 18.00 and 15.45 for $I, J$ and $K_{\mathrm{S}}$ respectively.

\section{Results and discussion}

If we take $K=15^{\mathrm{m}} 5$ as the noise level and intrinsic colours typical of YSOs or protostars we can conclude, that the object is enshrouded in a dust cloud that produces a minimum of $A_{V}=6^{\mathrm{m}}$. However, such a value is more likely a lower limit. As most of the stars in the field of the cloud are not visible in $I$ at all, we are unable to derive a statistically meaningful colour-colour diagram. But Cambrésy et al. (1997) have shown, that $K$ vs. $J-K$ diagrams can be applied for such kind of clouds to estimate the extinction. Using the number density and colour of the surrounding stars from the DENIS images rather provide an extinction of $\geq 10^{\mathrm{m}}$ for most of the stars (Fig. 5).

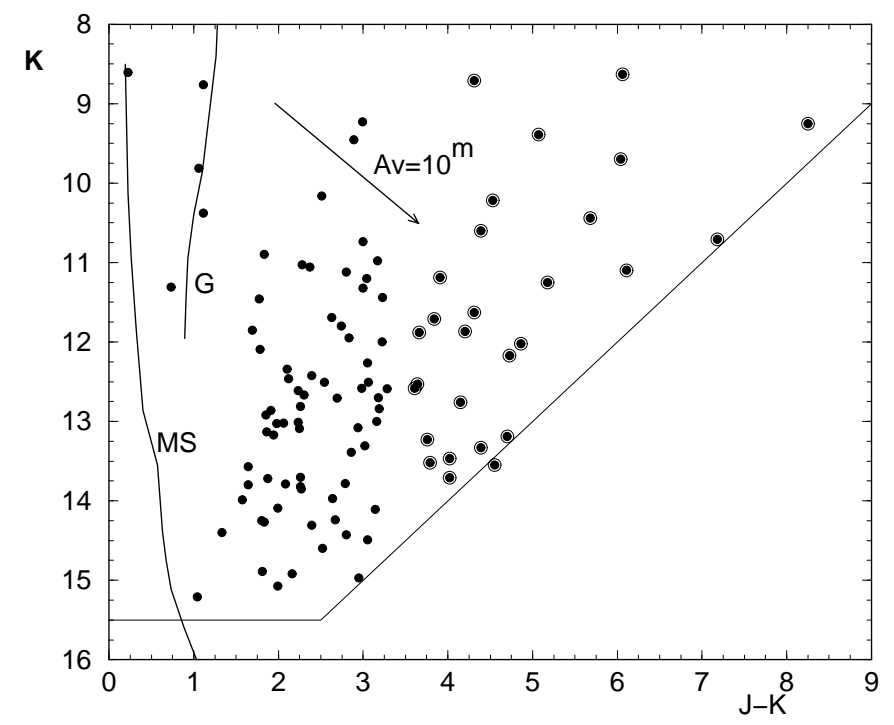

Fig. 5. The $K$ vs. $J-K$ diagram of the stars in the field. The loci of the main sequence and the giant branch for the estimated distance of $1.5 \mathrm{kpc}$ and the foreground extinction of $A_{V}=2^{\mathrm{m}}$ is indicated (see text). The arrow gives the reddening by an extinction of $A_{V}=10^{\mathrm{m}}$. The encirceled stars with ( $\mathrm{J}-$ $K)>3.5$ are clearly separated and are assumed to be YSO and T-Tauri candidates (see Cambrésy et al. 1998). The thin straight lines gives the limits due to the photometry

The four USNO stars (i.e. the stars of the "group") were assumed to be located on the front side of the cloud, and are thus not affected strongly by its extinction. We used USNO B $+\mathrm{R}$ and the DENIS $I, J$ and $K$ to classify those objects. They must be of luminosity class III; otherwise the cloud would be at distance, which is far below that of the bright foreground star HD 173955. 
Table 2. YSO and T-Tauri candidates from the $K$ vs. $J-K$ diagram. The positions are offsets in arcseconds from the position of the star HD 173955 as given in TYCHO2 $\left(18^{\mathrm{h}} 48^{\mathrm{m}} 22^{\mathrm{s}} .30-02^{\circ} 18^{\prime} 45^{\prime \prime}\right.$ 3, J2000)

\begin{tabular}{rrrrr}
\hline$N$ & $\Delta_{\alpha}$ & $\Delta_{\delta}$ & \multicolumn{1}{c}{$K$} & $J-K$ \\
\hline 1 & 11.2 & -183.2 & 10.44 & 5.68 \\
2 & 10.5 & -93.0 & 10.60 & 4.39 \\
3 & -11.8 & -165.1 & 12.76 & 4.15 \\
4 & -12.2 & -87.2 & 10.71 & 7.18 \\
5 & -14.6 & -33.5 & 11.10 & 6.11 \\
6 & -16.0 & 29.7 & 13.52 & 3.79 \\
7 & -23.0 & -189.2 & 11.88 & 3.66 \\
8 & -28.3 & -182.7 & 11.19 & 3.91 \\
9 & -34.2 & -190.0 & 10.22 & 4.53 \\
10 & -34.8 & -129.8 & 11.25 & 5.18 \\
11 & -40.0 & -171.4 & 12.58 & 3.61 \\
12 & -41.5 & -107.1 & 9.70 & 6.04 \\
13 & -52.3 & -74.8 & 13.47 & 4.02 \\
14 & -58.6 & 35.8 & 13.23 & 3.76 \\
15 & -64.5 & 26.8 & 11.71 & 3.84 \\
16 & -67.0 & -180.3 & 11.87 & 4.20 \\
17 & -75.1 & -100.7 & 8.71 & 4.31 \\
18 & -80.0 & -164.1 & 12.17 & 4.73 \\
19 & -83.6 & -4.4 & 9.39 & 5.07 \\
20 & -99.2 & 16.1 & 11.63 & 4.31 \\
21 & -127.6 & 11.2 & 12.02 & 4.86 \\
22 & -135.8 & 15.0 & 8.63 & 6.06 \\
23 & -147.9 & 24.5 & 13.33 & 4.39 \\
24 & -159.7 & 18.2 & 13.55 & 4.55 \\
25 & -161.2 & -168.2 & 12.53 & 3.64 \\
26 & -166.1 & -3.3 & 13.71 & 4.02 \\
27 & -173.4 & -49.1 & 13.19 & 4.70 \\
28 & -193.6 & -125.0 & 9.25 & 8.25 \\
\hline & & & & \\
1 & &
\end{tabular}

The five colours spanning a large range of wavelength allow, although the USNO photometry is not that accurate, to classify the stars as K3-6 III for 0825-13045391 and 0825-13044642 with a (foreground) extinction of $A_{V} \approx 2^{\mathrm{m}}$ and K7-M0 III for 0825-130414457 at an extinction of $A_{V} \approx 3^{\mathrm{m}}$. Only $0825-13043990$ does not give a unique solution. While it seems to be a late M giant by using the NIR colours alone, it appears to be of late $\mathrm{G}$ or early $K$ using the optical bands. This outcome might be due to the fact that the USNO colours are affected by the nebulosity around the object. On the $K$ vs. $J-K$ diagram it fits well the giant branch.

These data allow us to estimate a distance to the cloud of $1600 \pm 400 \mathrm{pc}$ and a foreground extinction of $A_{V} \approx 2 \pm 0.5$ magnitudes. This extinction is in good agreement with the known values for this region of the galactic plane from bright stars and average extinction models $\left(\ell=30^{\circ}, b=0.4^{\circ}\right)$. The higher extinction towards 0825-130414457 may be due to the cloud itself. This is supported by the comparison of the surface number density of stars in $J$ and $K$. Cambrésy et al. (1998) pointed out that stars beyond $A_{V} \approx 10^{\mathrm{m}}$ are likely YSO and T-Tauri candidates, embedded in circumstellar shells, while the stars with $1^{\mathrm{m}} \cdot 6<(J-K)<3^{\mathrm{m}} \cdot 2$ (which corresponds to a visual extinction range from 6 to $1^{\mathrm{m}}$ ) are either normal stars in the cloud or most probably background stars. This discrimination is supported also by the gap in the colour magnitude diagram at $J-K=3.5$. As the candidates are evenly distributed within the region, those stars are unlikely to be background stars of a cloud, which is embedding no stars at an optical depth $11^{\mathrm{m}} 0<A_{V}<16.0$.

Cambrésy et al. (1997) also found a good correlation of the 60 and $100 \mu \mathrm{m}$ flux of IRAS with the extinction maps obtained by NIR star counts. While this seems to be valid here too in the NW corner of the field, the SE corner gives contrasting results (see Fig. 6). It seems that the cloud here is more likely connected towards the west or north-west to a much larger complex around the grouping of radio sources [SG96] 30.50-0.29, [L89b] 30.50-0.29, F3R 1063 and the supernova remnant SNR 030.1+00.3 about 5 to $6^{\prime} \mathrm{NW}$ of our field here.

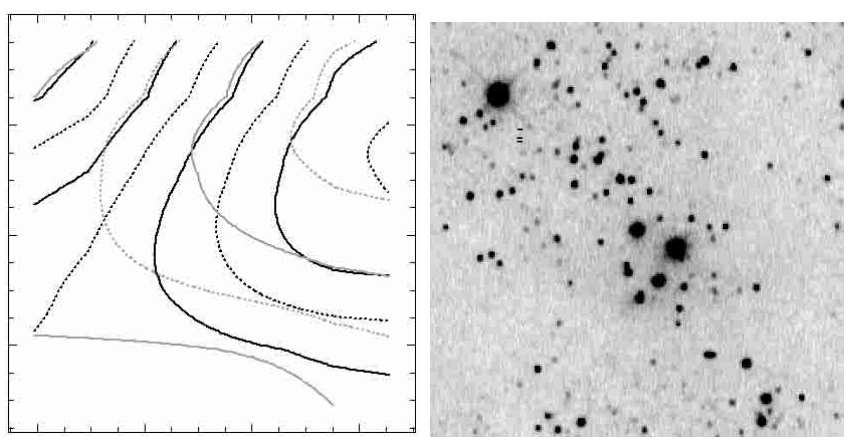

Fig. 6. Left: IRAS $100 \mu \mathrm{m}$ (black lines) and $60 \mu \mathrm{m}$ sky brightness contours (light grey) - scaled from zero to local maximum at 10 steps; right: the DENIS $J$ band image as in Fig. 4 . While the star counts suggest the highest extinction on the lower left side, the IRAS maps do not show any increase of the flux there

In conclusion, a new flare star and, as a consequence of subsequent DENIS observations, a couple of new young stellar objects in the region of this star have been detected. As the flair star was not detected in the NIR, it has to be strongly obscured by the cloud and its circumstellar material. The absolute magnitude of the flair have to be higher than those in the Plejades. This (statistically) gives a very young age of the cluster (Pigatto 1990; Mnatsakanian \& Mirzoyan 1990). We intend to monitor this star forming region.

Acknowledgements. This project was supported by the Austrian FWF project P11675-AST. We thank the DENIS consortium (PI N. Epchtein, Nice, F) for being able to do these additional observations. The DENIS project is partly funded by the European Commission grants. It is also supported, in France by the INSU, CNRS, in Germany by the 
State of Baden-Würtemberg, in Spain by the DGICYT, in Italy by the CNR, in Austria by the Fonds zur Förderung der wissenschaftlichen Forschung und Bundesministerium für Wissenschaft und Forschung, in Brazil by the FAPESP, and in Hungary by an OTKA grant and an ESO C \& EE grant.

\section{References}

Ambartsumian, V. A. 1990, IAUS, 137, 163

Cambrésy, L., Copet, E., Epchtein, N., et al. 1998, A\&A, 338, 977

Cambrésy, L., Epchtein, N., Copet, E., et al. 1997, A\&A, 324, L5

Epchtein, N., de Batz, B., Capoani, L., et al. 1997, The ESO Messenger, 87, 27

Epchtein, N., Deul, E., Derriere, S., et al. 1999, A\&A, 349, 296
Fouqué, P., Chevallier, L., Cohen, M., et al. 2000, A\&AS, 141, 313

Hartl, H., \& Weinberger, R. 1987, A\&AS, 69, 519

Hoessel, J. G., Elias, J. H., Huchra, J. P., \& Wade, R. A. 1979, PASP, 91, 41

Kimeswenger, S., Kerber, F., Roth, M., et al. 1998, A\&A, 332, 300

Kwok, S., Polk, K., \& Bidelman, W. P. 1997, ApJS, 112, 557

Monet, D., Bird, A., Canzian, B., et al. 1998, A Catalogue of Astrometric Standards, U.S. Naval Observatory, Flagstaff

Mnatsakanian, M. A., \& Mirzoyan, A. L. 1990, IAUS, 137, 113 Pigatto, L. 1990, IAUS, 137, 117

Saurer, W., Seeberger, R., \& Weinberger, R. 1997, A\&AS, 126, 247

Szymczak, M., Hrynek, G., \& Kus, A. J. 2000, A\&AS, 143, 269

Weinberger, R. 1991, IBVS, 3685 